\title{
Sensory Evaluation of Pearl Millet based Snack Food (Kharodi) using Fuzzy Logic
}

\author{
S.B. Solanke ${ }^{1}$, R.V. Jaybhaye ${ }^{2 *}$ and S.B. Jadhav ${ }^{3}$ \\ ${ }^{1}$ Department of Process \& Food Engineering, COAET, VNMKV, Parbhani-431402, India \\ ${ }^{2}$ Department of Agril. Engineering, Nanded Road, College of Agriculture, \\ Latur - 413512 (M.S.), India \\ ${ }^{3}$ Department of Agril. Engineering, College of Agriculture, Ambajogai, India \\ *Corresponding author
}

\begin{tabular}{l} 
Ke y w or d s \\
Sensory evaluation, \\
$\begin{array}{l}\text { Fuzzy logic, } \\
\text { Triplets, Similarity } \\
\text { values }\end{array}$ \\
\hline Article Info \\
\hline $\begin{array}{l}\text { Accepted: } \\
16 \text { March } 2018 \\
\text { Available Online: } \\
10 \text { April } 2018\end{array}$ \\
\hline
\end{tabular}

\section{Introduction}

Millets are important staples worldwide and apart from health benefits, they are also good source of energy, protein, vitamins and minerals (Rao, 1986). But their use in the diet is limited because of difficulty in processing and grittiness (Malleshi, 1986). Sensory evaluation comprises a set of techniques for accurate measurement of human responses to foods and minimizes the potentially biasing effects of brand identity and other information influences on the consumer perception. Sensory evaluation is an important tool in food industry. Fuzzy logic is an important tool by which vague and imprecise data can be analyzed and important conclusions regarding acceptance, rejection, ranking, strong and weak attributes of food can be drawn (Shinde and Pardeshi, 2014). In fuzzy modeling, linguistic variables (e.g., not satisfactory, good, excellent etc.) are used for developing 
relationship between independent (e.g. color, aroma, taste, mouthfeel, convenience etc.) and dependent (e.g. acceptance, rejection, ranking, strong and weak attributes of food) variables (Das, 2005; Routray and Mishra, 2011). As against the nine point hedonic scale (BIS, 1971), the linguistic responses of judges can be converted by fuzzy analysis into numerical values for comparison.

Fuzzy sets can be used for analysis of sensory data instead of average scores to compare the sample's attributes (Lincklaen et al., 1989; Kavdir and Gayer, 2003). Fuzzy sets theory introduced by Zadeh (1965), which allows uncertain phenomena to be treated mathematically. Chen et al., (1988) developed a model for the analysis of sensory data. Zhang and Litchfield (1991) developed a fuzzy comprehensive model for ranking of foods and developing new food products.

Ranking of food samples and their quality attributes are based on triplets associated with sensory scales, triplets for sensory score, triplets for sensory score of quality attributes, triplets for relative weightage of quality attributes, triplets for overall sensory score, values of membership function of standard fuzzy scale, values of overall membership function of sensory scores on standard fuzzy scale, similarity values, quality attributes ranking in general.

This method has been successfully applied for mango drinks (Jaya and Das, 2003), dahi powder (Routray and Mishra, 2011), instant green tea powder (Sinija and Mishra, 2011) and soy paneer (Uprit and Mishra, 2002). Very few researchers have applied fuzzy logic for sensory analysis of RTE dried snack foods.

Therefore, in the present study it was attempted to conduct a sensory analysis using fuzzy logic in order to analyze the acceptability of Kharodi samples.

\section{Materials and Methods}

Preparation of product samples and sensory evaluation

Kharodi is a sun dried RTE traditional snack food prepared from pearl millet or sorghum grits with other ingredients like garlic, chili powder, salt, oil after cooking in water and subsequent sun drying. In the present investigation $0.5 \mathrm{~kg}$ pearl millet ingredients were cooked in open pan and the cooked product was dried in hot air dryer in the form of small chunks. The process parameters were optimized in terms of water level $(1980 \mathrm{ml})$, cooking time (20 min), DT drying temperature $\left(65^{\circ} \mathrm{C}\right)$ and drying time $(8.8 \mathrm{~h})$.

Three samples $\left(S_{1}, S_{2}\right.$ and $\left.S_{3}\right)$ were prepared at optimum process parameters by taking product without adding sesame as sample $S_{1}$, with sesame as sample $S_{2}$ and fried sample with sesame as $\mathrm{S}_{3}$. Sensory characteristics of Kharodi were evaluated by a panel of judges and the responses of the judges were calculated in terms of numerical values. For sensory evaluation of the samples a panel of twenty judges including 10 males and 10 females in the age group of 20 to 50 years (Ranganna, 1987; Singh et al., 2012) belonging to faculty and research scholars of the department were selected on good health, average sensitivity, interests in sensory evaluation and familiarity with snack foods. The data obtained from subjective evaluation of three Kharodi samples were analyzed by using fuzzy logic.

The panelists were asked to indicate their preference for each sample based on the selected quality attributes of color, flavor, taste and mouthfeel (texture). As the crispness of product is perceived through a combination of tactile, kinesthetic, auditory sensations (Heidenreich et al., 2004) and also associated with rapid drop of force during mastication 
process (Vincent, 1998), texture of Kharodi was tested in terms of mouthfeel. Judges were familiarized with the definitions of the quality attributes of RTE snack food before sensory evaluation. They were asked to take two or three pieces of samples before testing them and give the score for flavor first in the score sheet. Also, they were advised to rinse their mouth with lukewarm water between the testing of each sensory character (Das, 2005; Sinija and Mishra, 2011) and between testing the consecutive samples (Jaya and Das, 2003).

Judges were instructed to give tick mark to appropriate respective fuzzy scale factor for each of the quality attributes of the sample after tasting it. The samples were rated as "Not satisfactory", "Fair", "Medium", "Good" and "Excellent". Judges were also instructed to give rank to quality attributes of Kharodi snack food in general (Shinde et al., 2016) by giving tick mark to the respective scale factors, viz. "Not at all important", "Somewhat important", "Important", "Highly important" and "Extremely important". The set of observations were analyzed using Fuzzy analysis of sensory scores.

\section{Fuzzy modeling of sensory data}

The major steps involved in the fuzzy modeling of sensory evaluation are (1) calculation of overall sensory scores of Kharodi snack food samples in the form of triplets (Fig. 1); (2) estimation of membership function on standard fuzzy scale (Fig. 2); (3) computation of overall membership function on standard fuzzy scale (Fig. 3); (4) estimation of similarity values and ranking of the Kharodi samples; and (5) quality attribute ranking of Kharodi samples in general.

A program in MATLAB 7.0 software was developed for the calculation of all the above mentioned steps (Das, 2005; McGarrity, 2008).

\section{Triplets associated with five point sensory scale}

The obtained sensory scores were converted into a set of three numbers, called 'triplet' on five point scale. Kharodi snack food samples and quality attributes were assigned fuzzy membership on a five point sensory scale (Das, 2005). The distribution pattern of five point sensory scale is poor/not at all important, fair/somewhat important, medium/important, good/highly important and excellent/ extremely important as shown in Figure 1.

Set of three numbers known as "triplet" is used to represent triangular membership function on five point scale where triangle 'abc' represents membership function for poor/not at all important category, triangle 'ade' represents distribution function for fair/somewhat important category, etc. Table 1 shows 'triplets' associated with five point sensory scales.

First number of the triplet denotes the value of abscissa at which the value of membership function is one. Second and third number of the triplet indicates the distance to left and right respectively of the first number where the membership function is zero (Routray and Mishra, 2011).

The triplet for a particular quality attribute of given sample can be obtained from the sum of sensory scores, triplets associated with five point sensory scale and the number of judges. For example, the colour attribute of a sample, when total number of judges were 20 and out of the total 20 judges, three judges gave 'Fair' score, six judges gave the score as 'Medium', fourteen gave 'Good' and seven gave 'Excellent'; the triplets for the sensory scores of colour can be calculated by Eq. 1 .

$C S_{1}=\frac{0(0025)+5(252525)+8(502525)+5(752525)+2(100250)}{20}$ 
Triplets for each quality attribute of all the samples and quality attributes of Kharodi samples in general were obtained as per eq. 1 . Similarly, from the calculated values of triplets for quality attributes of Kharodi samples in general, the triplets for relative weightage of quality attributes $\left(\mathrm{Q}_{\mathrm{Rel}}\right)$ were also calculated.

The 'Relative weightage of the quality attribute' for color, flavor, taste and mouthfeel were defined as: $\mathrm{QC}_{\text {rel }}=\mathrm{QC} / \mathrm{Q}_{\text {sum }}$, flavor: $\mathrm{QF}_{\text {rel }}=\mathrm{QF} / \mathrm{Q}_{\text {sum }}$, taste: $\mathrm{QT}_{\text {rel }}=\mathrm{QT} / \mathrm{Q}_{\text {sum }}$ and for mouthfeel: $\mathrm{QM}_{\text {rel }}=\mathrm{QS} / \mathrm{Q}_{\text {sum }}$, where $\mathrm{Q}_{\text {sum }}$ is the sum of first digit of triplets of all quality attributes in general.

Triplets for overall sensory scores of Kharodi samples

The triplets for overall sensory scores of snack food samples were calculated using eq. 2 , in which triplet for sensory score for each quality attribute was multiplied with the triplet for relative weightage of that particular attribute and the sum of resultant triplet values for all attributes was taken.

$$
\begin{aligned}
& \operatorname{SOS}_{1=} \operatorname{CS}_{1} \times Q C_{r e l}+F S_{1} \times Q F_{r e l} \\
& +T S_{1} \times+Q T_{r e l}+M S_{1} \times Q M_{r e l}
\end{aligned}
$$

Where, $C S_{1}, F S_{1}, T S_{1}$ and $M S_{1}$ represents the triplets corresponding to the colour, flavor, taste and mouthfeel of sample one and $Q C_{\text {rel }}$, $Q F_{\text {rel }}, Q T_{\text {rel }}$ and $Q M_{\text {rel }}$ denotes the triplets corresponding to the relative weightage of quality attributes of Kharodi samples in general. Using similar equations the overall sensory scores for all samples were calculated. The multiplication of triplet $(\mathrm{a} b \mathrm{c}$ ) with triplet (d e f) was done by applying a rule as given in Eq. 3.

$(a b c) \times(d e f)=(a \times d a \times e+d \times b a \times f+d$ $\times c)(3)$

\section{Membership function for standard fuzzy scale}

The triplets obtained by Five Point scale are converted into Six Point sensory scale referred to as Standard Fuzzy scale. The triangular distribution pattern of sensory scales using symbols $F_{1}, F_{2}, F_{3}, F_{4}, F_{5}$ and $F_{6}$ is given in Figure 2. Membership function of each of the sensory scales follows triangular distribution pattern where maximum value of membership function is one.

The values of fuzzy membership function lie between 0 and10. Therefore, values of $F_{1}$ through $\mathrm{F}_{6}$ are defined by a set of 10 numbers as given in Eq. 4.

$\mathrm{F}_{1}=(1,0.5,0,0,0,0,0,0,0,0)$

$F_{2}=(0.5,1,1,0.5,0,0,0,0,0,0)$

$\mathrm{F}_{3}=(0,0,0.5,1,1,0.5,0,0,0,0)$

$\mathrm{F}_{4}=(0,0,0,0,0.5,1,1,0.5,0,0)$

$\mathrm{F}_{5}=(0,0,0,0,0,0,0.5,1,1,0.5)$

$\mathrm{F}_{6}=(0,0,0,0,0,0,0,0,0.5,1)(4)$

\section{Overall membership function of sensory scores on standard fuzzy scale}

The overall quality of the Kharodi samples was linked to the standard fuzzy scale. The overall quality, as expressed by a triplet $(a, b$, c) was represented by a triangle $\mathrm{ABC}$, shown in Figure 3.

The graphical representation of membership function of a triplet $(a, b, c)$ is given in Figure 3 . The figure shows that for a triplet $(a, b, c)$, when the value of abscissa is $a$, value of membership function is 1 and when it is less than $a-b$ or greater than $a+c$, the value is 0 . For a given value of $x$ on abscissa, value of membership function $B_{x}$ can be expressed by similar triangles as given in eq. 5 .

$B_{x}=\frac{x-(a-b)}{b}$ for $(a-b)<x<a$ 
$B_{x}=\frac{(a+c)-x}{c}$ for $a<x<(a+c)$

$B_{x}=0$ for $x<(a-b)$ and $x>(a+c)$

For overall sensory quality of each of the samples and for quality attributes of Kharodi in general, the value of membership function $B_{x}$ at $x=0,10,20,30,40,50,60,70,80,90$ and 100 were found out from eq. 5. This membership function value of samples and quality attributes in general on standard fuzzy scale was given as set of 10 numbers which are '(maximum value of $B_{x}$ at $0<x<10$ ), (maximum value of $B_{x}$ at $10<x<20$ ), (maximum value of $B_{x}$ at $20<x<30$ ), (maximum value of $B_{x}$ at $30<x<40$ ), (maximum value of $B_{x}$ at $40<x<50$ ), (maximum value of $B_{x}$ at $50<x<60$ ), (maximum value of $B_{x}$ at $60<x<70$ ), (maximum value of $B_{x}$ at $70<x<80$ ), (maximum value of $B_{x}$ at $80<x<90$ ), (maximum value of $B_{x}$ at $90<x<100$ )'.

\section{Similarity values and ranking of snack food samples}

After getting the B values for each sample and quality attribute in general on standard fuzzy scale as a set of 10 values, the similarity values for each triplet of samples and quality attributes were obtained by the eq. 6. (Sinija and Mishra, 2011).

$S_{m}(F, B)=\frac{F \times B^{r}}{\operatorname{Max}\left(F \times F^{\prime} \text { and } B \times B^{\prime}\right)}$

Where, $S_{m}$ is the similarity value for the sample and quality attribute in general, $F \times B^{\prime}$ is the product of matrix $F$ with the transpose of matrix $B, F \times F^{\prime}$ is the product of matrix $F$ with the transpose of $F$ and $B \times B^{\prime}$ is the product of matrix $B$ with its transpose. For sample one similarity values will be $S_{m}\left(F_{1}\right.$, $\left.B_{1}\right), S_{m}\left(F_{2}, B_{1}\right), S_{m}\left(F_{3}, B_{1}\right), S_{m}\left(F_{4}, B_{1}\right), S_{m}$ $\left(F_{5}, B_{1}\right)$ and $S_{m}\left(F_{6}, B_{1}\right)$. The values were calculated using the rules of matrix multiplication.

Similarity values under the six categories of sensory scales were compared to find out the highest similarity value. The category corresponding to the highest similarity value was considered responsible for its quality. The overall quality of each of the samples was defined using above procedure. By combining the defined overall qualities of the samples as calculated by the above procedure, ranking of three samples and quality attributes in general was done by fuzzy comprehensive modeling (Zhang and Litchfield, 1991).

\section{Results and Discussion}

The sensory scores as given by the panel of judges' is shown in Table 2. The analysis of sensory attributes of Kharodi samples was conducted using fuzzy logic (Das, 2005).

\section{Triplets for sensory quality of Kharodi samples}

Table 2 shows the sum of sensory scores according to preferences given by the judges for the Kharodi samples prepared from the developed dried product without sesame (Sample $\mathrm{S}_{1}$ ), with sesame (Sample $\mathrm{S}_{2}$ ) and fried sample (Sample $\mathrm{S}_{3}$ ).

Triangular membership function distributions of sensory scales were given by 'triplets', which are sets of three numbers. Calculation of sensory quality attributes of Kharodi samples in triplets form (Column 6 of Table 2) was done from (i) sum of sensory scores given by judges during sensory evaluation, (ii) triplets associated with the sensory scale (Table 1) and (iii) number of judges who gave tick mark under particular head on sensory scale (Table 2). The triplet for a particular quality attribute of given sample was obtained from the sum of sensory scores, triplets 
associated with five point sensory scale and the number of judges. The results of calculations of triplets for three samples under sensory evaluation are given in Table 2.

Triplets for importance of quality attributes in general and relative weightage

The sensory scores given by judges and the triplets for quality attributes in general of Kharodi are given in Table 3. The triplets for individual preference to the importance of quality attributes of Kharodi in general were calculated using the eq. 1 similar to triplets for three samples. Thus, the triplets for judges' preference to importance of quality attributes, viz., color $(\mathrm{QC})$, flavor $(\mathrm{QF})$, taste $(\mathrm{QT})$ and mouthfeel $(\mathrm{QM})$ were as given in Table 3 and were denoted as eq. 7.

It is necessary to bring the value of the first digit of overall sensory score between 0 and 100. In order to do this, the values of triplets for quality attributes in Table 3 were reduced by a factor $1 / Q_{\text {sum }}$, where, $Q_{\text {sum }}$ is the sum of the first digit of all the triplets which was calculated as given in eq. 8 (Singh et al., 2012).

$\mathrm{Q}_{\text {sum }}=51.25+57.50+97.50+88.75=295.0(7)$

The triplets for relative weightage of the quality attributes for color, flavor, taste and mouthfeel were calculated as:

$\mathrm{QCrel}=(0.066,0.095,0.095)$

QFrel $=(0.220,0.095,0.095)$

QTrel $=(0.373,0.095,0.095)$

$\mathrm{QMrel}=(0.339,0.095,0.043)(8)$

Overall sensory scores as triplets for Kharodi samples

The overall sensory scores triplets for Kharodi samples were calculated as the sum of the products of the triplets for Kharodi samples and relative weightages given in Table 2 and eq. 8 using eq. (2) and triplet multiplication rule given in eq. (3).

The calculated values of triplets for overall sensory scores of Sample $1\left(S O^{S_{1}}\right)$, Sample 2 $\left(\mathrm{SO}^{S_{2}}\right)$ and Sample $3\left(\mathrm{SO}^{S_{3}}\right)$ are as follows:

$\operatorname{SOS}_{1}=\left(\begin{array}{lll}52.5155 & 44.282 & 42.0373\end{array}\right)$

$\operatorname{SOS}_{2}=\left(\begin{array}{lll}75.1658 & 52.719 & 40.4711\end{array}\right)$

$\operatorname{SOS}_{3}=\left(\begin{array}{llll}88.7392 & 57.863 & 37.3181\end{array}\right) \quad \ldots(9)$

From the first digit values in triplets for three samples it can be inferred that people have preferred sample 3 and sample 2 over sample 1 . As sample 2 and sample 3 were prepared by adding sesame, it implies that sesame improves the sensory quality of Kharodi.

\section{Membership function of overall sensory scores on standard fuzzy scale}

Overall membership functions for triplets of Sample 1, Sample 2 and Sample 3 on standard fuzzy scale were calculated by using the eq. 5 and values in eq. 9. These values $\left(B_{1}\right.$ to $\left.B_{10}\right)$ are called as fuzzy membership values and are given in eq. 10 as:

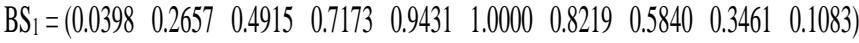

$\mathrm{BS}_{2}=\left(\begin{array}{llllllllll}0 & 0 & 0.1432 & 0.3329 & 0.5226 & 0.7123 & 0.9020 & 1.0000 & 0.4351 & 0.1880\end{array}\right)$

$\mathrm{BS}_{3}=\left(\begin{array}{llllllllll}0 & 0 & 0 & 0.1576 & 0.3305 & 0.5033 & 0.6761 & 0.8489 & 1.0000 & 0.9662\end{array}\right)$

\section{Similarity values and ranking of Kharodi samples}

The similarity values were calculated by comparing the membership function values $\left(\mathrm{BS}_{1}, \mathrm{BS}_{2}, \mathrm{BS}_{3}\right)$ of three samples in eq. 10 with their corresponding F1 to F6 values in eq. 4. 
Table.1 Triplets associated with five point sensory scale

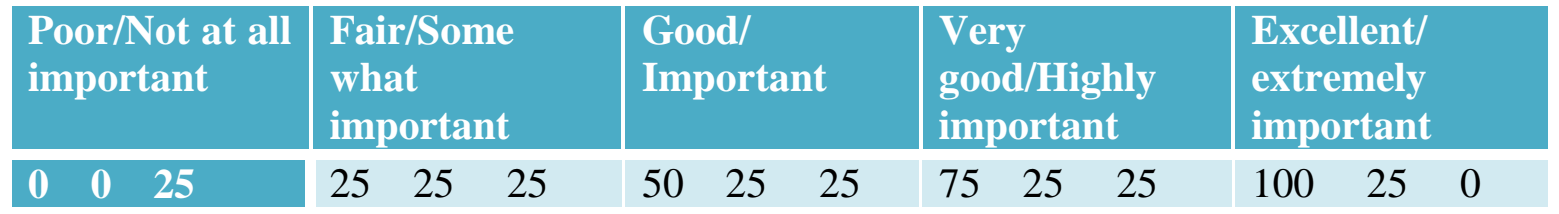

Table.2 Sum of sensory scores in terms of preference given by judges and corresponding triplets for sensory quality of Kharodi samples

\begin{tabular}{|c|c|c|c|c|c|c|}
\hline $\begin{array}{l}\text { Sensory } \\
\text { quality } \\
\text { attributes }\end{array}$ & Poor & Fair & Medium & Good & Excellent & $\begin{array}{l}\text { Triplets for Sensory Quality of } \\
\text { Samples }\end{array}$ \\
\hline \multicolumn{7}{|l|}{ Colour } \\
\hline Sample 1 & 0 & 5 & 8 & 5 & 2 & $\mathrm{CS} 1=\left(\begin{array}{lll}55.00 & 16.00 & 22.50\end{array}\right)$ \\
\hline Sample 2 & 0 & 3 & 5 & 8 & 4 & $\mathrm{CS} 2=\left(\begin{array}{lll}66.25 & 25.00 & 20.00\end{array}\right)$ \\
\hline Sample 3 & 0 & 0 & 0 & 11 & 9 & $\mathrm{CS3}=\left(\begin{array}{lll}86.25 & 25.00 & 13.75\end{array}\right)$ \\
\hline \multicolumn{7}{|l|}{ Flavour } \\
\hline Sample 1 & 0 & 6 & 5 & 8 & 1 & FS1 $=\left(\begin{array}{lll}55.00 & 25.00 & 23.75\end{array}\right)$ \\
\hline Sample 2 & 0 & 2 & 5 & 10 & 3 & $\mathrm{FS} 2=\left(\begin{array}{lll}67.5 & 25.00 & 21.25\end{array}\right)$ \\
\hline Sample 3 & 0 & 0 & 2 & 13 & 5 & $\mathrm{FS3}=\left(\begin{array}{lll}78.75 & 25.00 & 18.75\end{array}\right)$ \\
\hline \multicolumn{7}{|l|}{ Taste } \\
\hline Sample 1 & 0 & 2 & 9 & 8 & 1 & $\mathrm{TS} 1=\left(\begin{array}{lll}57.50 & 25.00 & 23.75\end{array}\right)$ \\
\hline Sample 2 & 0 & 0 & 3 & 7 & 10 & $\mathrm{TS} 2=\left(\begin{array}{lll}83.75 & 25.00 & 12.50\end{array}\right)$ \\
\hline Sample 3 & 0 & 0 & 0 & 3 & 17 & $\mathrm{TS3}=\left(\begin{array}{lll}96.25 & 25.00 & 3.75\end{array}\right)$ \\
\hline \multicolumn{7}{|l|}{ Mouthfeel } \\
\hline Sample 1 & 1 & 7 & 7 & 5 & 0 & $M S 1=\left(\begin{array}{lll}45.00 & 23.75 & 25.00\end{array}\right)$ \\
\hline Sample 2 & 0 & 3 & 3 & 7 & 7 & MS2 $=\left(\begin{array}{lll}72.5 & 25.00 & 16.25\end{array}\right)$ \\
\hline Sample 3 & 1 & 0 & 0 & 6 & 13 & $M S 3=\left(\begin{array}{lll}87.50 & 23.75 & 8.75\end{array}\right)$ \\
\hline
\end{tabular}

Table.3 Sum of sensory scores in terms of preferences given by judges and triplets for Importance of quality attributes of Kharodi in general

\begin{tabular}{|c|c|c|c|c|c|c|}
\hline $\begin{array}{l}\text { Quality } \\
\text { attributes }\end{array}$ & NI & SI & $\mathbf{I}$ & HI & EI & Triplets for Quality attributes \\
\hline Colour & 1 & 9 & 13 & 6 & 1 & $\mathrm{QC}=\left(\begin{array}{lll}51.25 & 25.00 & 25.00\end{array}\right)$ \\
\hline Flavour & 0 & 3 & 10 & 14 & 3 & $\mathrm{QF}=\left(\begin{array}{lll}57.50 & 25.00 & 25.00\end{array}\right)$ \\
\hline Taste & 0 & 0 & 6 & 10 & 15 & $\mathrm{QT}=\left(\begin{array}{lll}97.50 & 25.00 & 25.00\end{array}\right)$ \\
\hline \multirow[t]{2}{*}{ Mouthfeel } & 0 & 0 & 6 & 14 & 10 & $\mathrm{QM}=\left(\begin{array}{lll}88.75 & 25.00 & 11.25\end{array}\right)$ \\
\hline & & & & & & Qsum= 295.0 \\
\hline
\end{tabular}

NI - Not at all important, SI - Somewhat important, I - Important, HI - Highly important, EI - Extremely important 
Table.4 Similarity values of Kharodi samples and their ranking

\begin{tabular}{|l|c|c|c|}
\hline Sensory scale & $\begin{array}{c}\text { Sample1 } \\
\text { (without sesame) }\end{array}$ & $\begin{array}{c}\text { Sample 2 } \\
\text { (with sesame) }\end{array}$ & $\begin{array}{c}\text { Sample 3 } \\
\text { (fried sample) }\end{array}$ \\
\hline Poor & 0.0446 & 0 & 0 \\
\hline Fair & 0.2937 & 0.1519 & 0.0225 \\
\hline Medium & $\mathbf{0 . 6 2 2 4}$ & 0.5132 & $\mathbf{0 . 2 1 1 4}$ \\
\hline Good & 0.5377 & $\mathbf{0 . 9 9 0 2}$ & $\mathbf{0 . 6 5 0 7}$ \\
\hline Very good & 0.3609 & 0.7920 & $\mathbf{0 . 7 6 3 1}$ \\
\hline Excellent & 0.0727 & 0.1622 & $\mathbf{0 . 4 1 9 0}$ \\
\hline Ranking & JII & II & I \\
\hline
\end{tabular}

Table.5 Similarity values of quality attributes of Kharodi in general

\begin{tabular}{|l|c|c|c|c|}
\hline Scale factors & Colour & Flavor & Taste & $\begin{array}{c}\text { Mouthfeel } \\
\text { (Texture) }\end{array}$ \\
\hline Not at all important & 0.0000 & 0.0000 & 0.0000 & 0.0000 \\
\hline Somewhat important & 0.0522 & 0.0566 & 0.0000 & 0.0000 \\
\hline Important & $\mathbf{0 . 9 4 9 3}$ & 0.5660 & 0.000 & $\mathbf{0 . 0 0 0 0}$ \\
\hline Very important & 0.8294 & $\mathbf{0 . 9 4 3 3}$ & 0.0949 & $\mathbf{0 . 2 3 0 0}$ \\
\hline Highly important & 0.2119 & 0.3962 & $\mathbf{0 . 9 4 9 3}$ & $\mathbf{0 . 8 8 6 0}$ \\
\hline Extremely important & 0.0000 & 0.0188 & 0.8544 & $\mathbf{0 . 6 1 3 3}$ \\
\hline Ranking & IV & III & I & II \\
\hline
\end{tabular}

Fig.1 Triplets associated with five point sensory scale

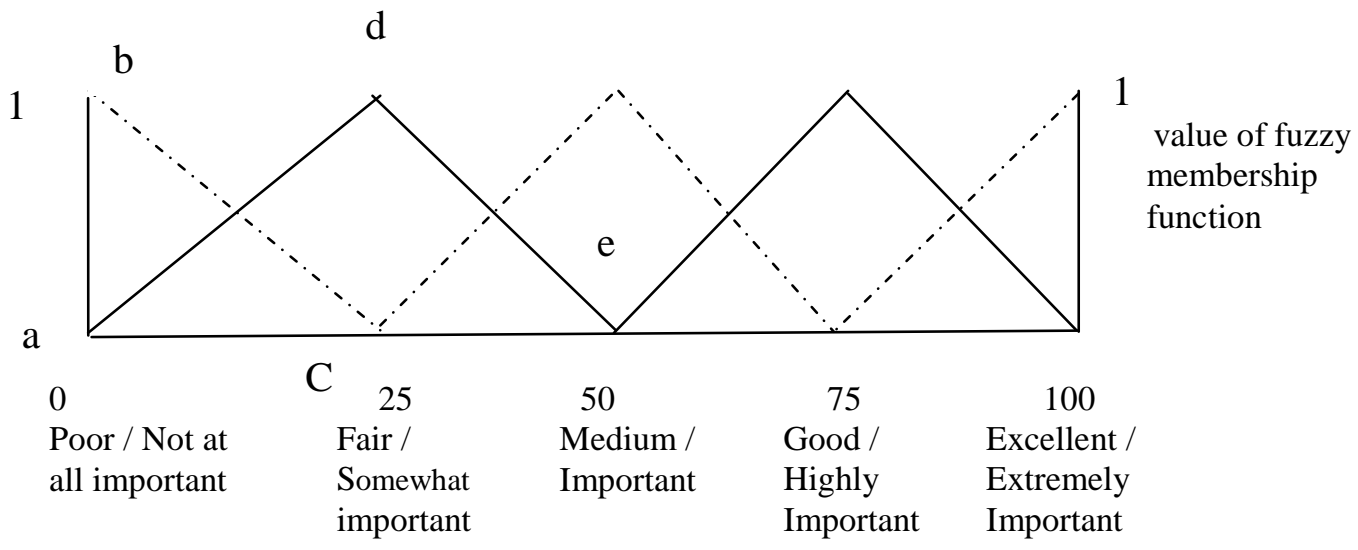


Fig.2 Standard fuzzy scale

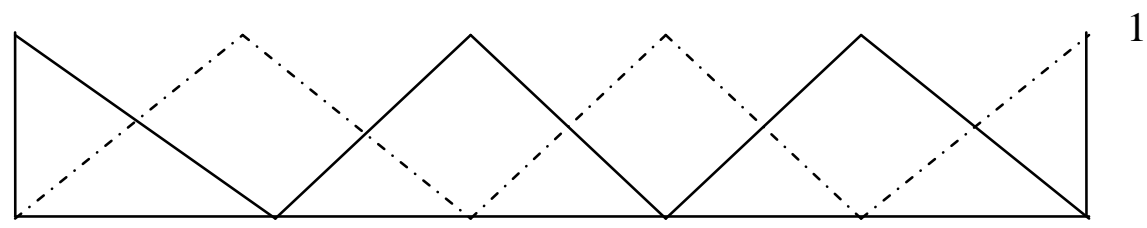

\begin{tabular}{lcccll}
\multicolumn{1}{c}{0} & 20 & 40 & 60 & 80 & 100 \\
\multicolumn{1}{c}{$\mathrm{F}_{1}$} & $\mathrm{~F}_{2}$ & $\mathrm{~F}_{3}$ & $\mathrm{~F}_{4}$ & $\mathrm{~F}_{5}$ & $\mathrm{~F}_{6}$ \\
Not & Fair / & Satisfactory & Good / & Very good & Excellent \\
satisfactory / & Somewhat & / Necessary & Important & / Highly & / \\
Not at all & necessary & & Important & Important & Extremely \\
necessary & & & & & important
\end{tabular}

Fig.3 Graphical view of overall sensory score as triangle $\mathrm{ABC}$ and triplet (A, B, C)

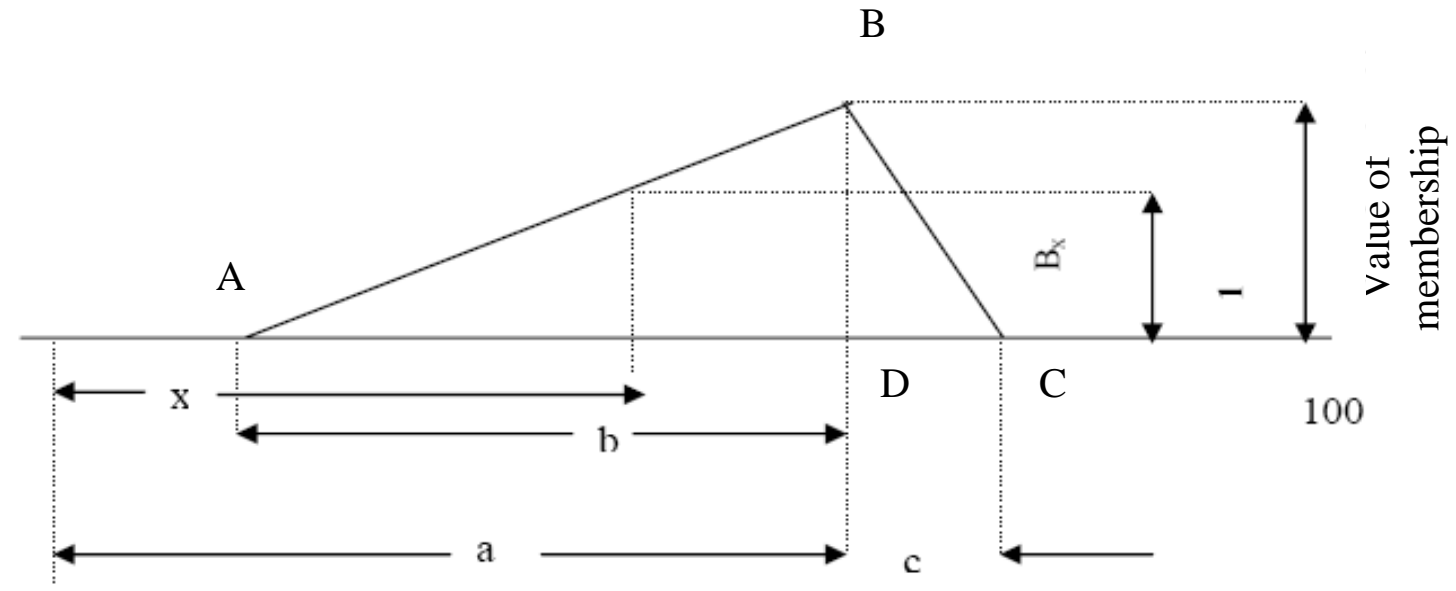

The ranking of samples was done on the basis of obtained similarity values under Poor $(F 1)$, Fair (F2), Medium (F3), Good (F4), Very good $(F 5)$ and Excellent (F6) categories. The similarity values for all the three samples under different scale factors are presented in Table 4.

From the Table 4, it can be seen that, for Sample 1 the highest similarity value lies in the category 'medium' (0.6224). For Sample 2, highest similarity value is obtained under the category 'good' and for Sample 3 it is under the category 'very good'. On comparison of highest similarity values, their ranking was done as
Sample $3>$ Sample $2>$ Sample 1, where Sample 3 is fried sample with sesame addition, Sample 2 is with sesame addition and Sample 1 is without sesame addition. Thus, it indicates that fried Kharodi sample was preferred by judges. Also, the score of sample 2 was very close to that of fried sample under the category of 'very good'. Therefore, present method of adding sesame in Kharodi improves the sensorial property of Kharodi prepared from pearl millet flour; hence it is value added process. Similar ranking under the category 'very good' was obtained by Shinde et al., (2016) for RTE snack food prepared from 
composite minor millet flour with and without incorporation of sprouted soybean.

\section{Fuzzy Membership function of quality attributes in general on standard fuzzy scale}

Similar procedure as above was adopted for calculation and ranking of quality attributes (colour, flavor, taste and mouthfeel) of Kharodi in general. The triplets for importance of these quality attributes in general have been given in Table 3. Using the above method of computation and denoting $\mathrm{BC}, \mathrm{BF}, \mathrm{BT}$ and $\mathrm{BM}$ as overall sensory scores on standard fuzzy scale for membership function of colour, flavor, taste and mouthfeel respectively, we get,

$\mathrm{BC}=(0,0,0.15,0.55,0.95,1,0.65,0.25,0,0)$

$\mathrm{BF}=(0,0,0,0.30,0.70,1,0.90,0.50,0.10,0)$

$\mathrm{BT}=(0,0,0,0,0,0,0,0.30,0.70,1)$

$\mathrm{BM}=(0,0,0,0,0,0,0.25,0.65,1,0.89)(11)$

The similarity values of four quality attributes of the Kharodi snack food were calculated by eq. 6 using the F values in eq. 4 and B values in eq. 11. The computed values are given in Table 5 .

The results from Table 5 show that taste $(0.9493)$ is the highest quality attribute followed by mouthfeel $(0.8860)$ and these two quality attributes can be considered as highly important for RTE Kharodi in general. This is followed by flavour (0.9433) and colour (0.9493). The flavour and colour of Kharodi were rated under the category 'Very important' and 'Important' respectively.

The order of preference given by judges for quality attributes of Kharodi in general was Taste (highly important) > Mouthfeel (highly important) $>$ Flavour (very important) $>$ Colour (important). Among the quality attributes, taste was the strongest and colour was the weakest quality attribute for Kharodi which was similar to the preference reported by Mazumder et al.,
(2007) for dry snack food and Jaybhaye (2012) for puffed RTE barnyard millet snack food.

The similarity values of taste and mouthfeel are close to each other and are highly important for Kharodi pearl millet snack food. The close tie between two quality attribute indicates the wellestablished consumer choice and product. Thus, taste and mouthfeel are the highly important quality parameters; colour is the least important quality parameter as per the preferences of judges in this evaluation.

It can be concluded that the fuzzy logic analysis can be used for sensory evaluation of RTE snack food and the samples of Kharodi product can be ranked based on consumer preference. The results of sensory analysis also showed that the addition of sesame in cooked pearl millet grits improves the quality of Kharodi and is comparable with the sample after frying. Considering the importance of oil free food, Kharodi with sesame can be considered as suitable RTE snack food.

\section{Acknowledgement}

The authors acknowledge the financial help and support given by the Head of the department of Process and Food Engineering and the Associate Dean and Principal, College of Agricultural Engineering and Technology, VNMKV, Parbhani (M.S.), India.

\section{References}

BIS, 1971. Guide for sensory evaluation of foods. Optimum requirements IS: 6273 (Parts-II). New Delhi: Bureau of Indian Standards.

Chen, S.M. 1988. A new approach to handling fuzzy decision making. IEEE Transactions on Systems, Man and Cybernetics, 18: 1012-1016.

Das, H. 2005. Food Processing Operations Analysis. Asian Books Pvt. Ltd. New Delhi, pp. 357-402.

Heidenreich, S., Jaros, D., Rohm, R., and Ziems, A. 2004. Relationship between water activity and crispness of extruded 
rice crisps. J. of Texture Studies, 35:621633.

Jaya, S., and Das, H. 2003. Sensory evaluation of mango drinks using fuzzy logic. Journal of Sensory Studies, 18:163-176.

Jayebhaye, R.V. 2012. Development of hot air puffing machine for preparation of millet based RTE snack food. Unpublished Ph. $\mathrm{D}$ thesis, Agricultural and Food Engineering Department, IIT, Kharagpur (W.B.)-721302, India.

Kavdir, I., and Gayer, D.E. 2003. Apple grading using Fuzzy logic. Turkish Journal of Agriculture, 27: 375-382.

Lincklaen, W., Westenberg, S.D., Jong, D.A., Meel, J.F.A., Quadt, E., and Duin, R.P.W. 1989. Fuzzy set theory applied to product classification by a sensory panel. Journal of Sensory Studies, 4(1): 55-72.

Malleshi, N.G. 1986. Processing of small millets for food and industrial uses. In Proceeding of first international small millet workshop, Banglore, October 29 to November 2, 1986 (pp. 321 - 324). Oxford and IBH.

Mazumder, P., Roopa, B.S., and Bhattacharya, S. 2007. Textural attributes of a model snack food at different moisture contents. Journal of Food Engineering, 79:511-516.

McGarrity, S. 2008. Introduction to objectoriented programming in MATLAB $\AA$, Matlab Digest. www.mathworks.com.

Ranganna, S. 1987. Handbook of analysis and quality control for fruit and vegetables products, Tata McGraw Hill. Pp. 156 196.

Rao, M.A. 1986. Rheological properties of fluid foods. In M.A. Rao, and S.S.H. Rizvi (Eds.), Engineering properties of foods. New York: Marcel Dekker INC., pp. 1 48.
Routray, W., and Mishra, H.N. 2011. Sensory evaluation of different drinks formulated from dahi (Indian yogurt) powder using fuzzy logic. Journal of Food Processing and Preservation, doi:10.1111/j.17454549.2011.00545.

Shinde, K.J., and Pardeshi, I.L. 2014. Fuzzy logic model for sensory evaluation of commercially available jam samples. Journal of Ready to Eat Food, 01(02): 7884.

Shinde, K.J., Pardeshi, I.L., Pawar, S.G., and Gajakos, A.V. 2016. Applications of fuzzy logic technique in sensory evaluation of ready to eat foods. Journal of Ready to Eat Food, 03 (04): 45-50.

Singh, K.P., Mishra, A., and Mishra, H.N. 2012. Fuzzy analysis of sensory attributes of bread prepared from millet-based composite flours. $L W T$ - Food Science and Technology, 48 (02): 276- 282.

Sinija, V.R., and Mishra, H.N. 2011. Fuzzy analysis of sensory data for quality evaluation and ranking of instant green tea powder and granules. Food and Bioprocess Technology, 4: 408-416.

Uprit, S., and Mishra, H.N. 2002. Fuzzy multi attribute decision making approach and comparison of soya fortified paneer. Journal of Sensory Studies, 17(2): 163176.

Vincent, J. F. V. 1998. The quantification of crispness. Journal of Science of Food and Agriculture, 78: 162-168.

Zadeh, L. 1965. Fuzzy sets: Information and control, 8: 338-353.

Zhang, Q., and Litchfield, J.B. 1991. Applying fuzzy mathematics to product development and comparison. Food Technology, 45(7): 108-115.

\section{How to cite this article:}

Solanke, S.B., R.V. Jaybhaye and Jadhav, S.B. 2018. Sensory Evaluation of Pearl Millet based Snack Food (Kharodi) using Fuzzy Logic. Int.J.Curr.Microbiol.App.Sci. 7(04): 2144-2154. doi: https://doi.org/10.20546/ijcmas.2018.704.244 